\title{
DDT: Effects on maternal behavior
}

\author{
KAREN PAULSEN, VINCENT J. ADESSO and JCHN J. PORTER \\ University of Wisconsin-Milwaukee, Milwaukee, Wisconsin 53201
}

\begin{abstract}
To examine the hypothesis that DDT produces hyperexcitability which in turn affects maternal behavior, 14 rats were fed food containing concentrations of DDT. Results showed low DDT (50 ppm) animals exhibited significantly less nursing and greater rejection than the high DDT (100 ppm) and control $(0 \mathrm{ppm})$ groups. It was concluded that poor maternal behavior, and not only DDT ingestion, caused harm to neonates.
\end{abstract}

Although the physiological effects of DDT poisoning in animals have been examined, no unifying theory is available to explain the observed effects of dichlorodiphenyltrichloroethane (DDT) (Goodman \& Gilman, 1970; O'Brien, 1967). There have been few studies of the behavioral effects of DDT. One such study, Sobotka (1971) found that low DDT dosages of up to $25 \mathrm{mg} / \mathrm{kg}$ administered to rats increased motor activity and decreased habituation to an open-field situation. Sobotka suggested that DDT interferes with the central inhibitory system, thorough the weakening of the cholinergic system and release of formely inhibited motor activity.

One aspect of DDT-poisoning, that DDT ingested by a pregnant female may cause harm in fetal and natal stages, the later via the mother's milk, has been studied with conflicing results. Fahima, Bennett, and Hall (1970) administered DDT doses of 1,5 , or $25 \mathrm{mg} / \mathrm{kg}$ to three groups of nursing rats $24 \mathrm{~h}$ after delivery. The manipulation of DDT dosage level resulted in a neonate mortality rate over 21 days of $13 \%, 32 \%$, and $100 \%$, respectively, compared to $4 \%$ for controls. Naishtein and Leibovich (1971), investigating the effect of small doses of DDT on sexual function, found that the weight of newborn rats in all experimental groups was consistently lower than that of the control group for the 28 days of the study. They attribute this lag to the transmission of the pesticides from the mother to the young. Al-Hachim and Fink (1968) suggest that DDT delays the development of the CNS for a few weeks after birth.

There are data that are not consistent with the hypothesis that DDT ingested from the mother's milk causes neonate mortality. Henderson and Woolley (1969) found that three times as much DDT was required to reach LD50 for 10-day-old rats as compared to 60-day-old rats. In addition, Lu, Jessup, and Levallee (1965) has reported an LD50 greater than $4 \mathrm{~g} / \mathrm{kg}$ for day old rats, $437 \mathrm{mg} / \mathrm{kg}$ for 14 to 16 -day-old rats and $195 \mathrm{mg} / \mathrm{kg}$ for adult rats. Henderson and Woolley indicated that the immature rat is much more resistant to the effects of DDT due to a less efficient nervous

Karen Paulsen is now at Southern Illinois University.

Requests for reprints should be sent to Vincent $J$. Adesso, University of Wisconsin-Milwaukee, Department of Psychology, Milwaukee, Wisconsin 53201 . system both in that DDT is not absorbed from the blood and a brain less sensitive to DDT's lethal effects. In addition, an examination of the growth table for the pups in the aforementioned Naishtein and Leibovich (1971) study indicated that all animals weighed the same at birth but gradually became more and more disparate over days, possibly due, at least in part, to maternal behavior such as inferior nursing (Paulsen, Adesso, \& Porter, 1973).

Taken together these latter data suggest that it may be the behavior of the DDT-poisoned mothers that adversely affects the neonate and not direct ingestion of DDT through the mother's milk by the neonate. An experiment done at Patuxent Wildlife Research Center (Farney, 1971) found that DDT administered to hawks produced thin-shelled eggs as well as abnormal parental behavior such as devouring their eggs or young. Treon, Boyd, Berryman, Gosney, Hartman, Brown, and Coomer (1954) report that DDT did affect mortality during the suckling period while it had no significant effect on number of pregnancies, litter weight, or litter number in rats. Paulsen et al. (1973) found that rat mothers treated with DDT exhibited inferior nest building and retrieval, less nursing time, and more activity than a control animal. Mean weights for the DDT litters were less than those of the controls.

The present experiment was designed to determine whether ingestion of DDT by the neonate is the only cause of mortality or whether the hyperexcitability of DDT-poisoned mothers (Goodman \& Gilman, 1970; O'Brien, 1967) may cause poor maternal behavior, also leading to death of the newborns.

\section{METHOD}

\section{Subjects}

Twenty-four 90 to 100 day old, $250 \mathrm{~g} \mathrm{1-week-pregnant}$ primiparous albino rats were obtained from the Holtzman Company of Madison, Wisconsin. The subjects were randomly assigned to three groups of eight and fed dosages of DDT (20\% $\mathrm{o}^{\prime} \mathrm{p}^{\prime}$ and $80 \% \mathrm{p}^{\prime} \mathrm{p}^{\prime}$ form) dissolved in pure peanut oil mixed with milled rat food as follows: Group 0, 0 ppm; Group 50, $50 \mathrm{ppm}$; and Group $100,100 \mathrm{ppm}$ in $\mathrm{mg} / \mathrm{kg}$ body weight. Water was provided add lib and subjects were fed $10 \mathrm{~g}$ of the food mixture per day for 2 weeks' preparturition. Immediately post partum, all subjects were put on an ad-lib diet of Noyes pellets. 
Table 1

Individual Nursing Time* for Groups 0, 50, and 100 Over Weeks 1 and 2

\begin{tabular}{ccccc}
\hline $\begin{array}{c}\text { DDT } \\
\text { Dosage }\end{array}$ & Subject & Week 1 & Week 2 & Total \\
\hline \multirow{5}{*}{ 0/ppm } & 1 & 233 & 127.5 & 360.5 \\
& 2 & 239.5 & 156 & 395.5 \\
& 3 & 240.75 & 146.5 & 387.25 \\
& 5 & 237.5 & 200 & 437.5 \\
& 5 & 178.5 & 86.75 & 265.25 \\
& 7 & 200.25 & 162 & 362.25 \\
& 8 & 218 & 82 & 300 \\
$50 /$ ppm & 1 & 455.25 & 136.5 & 391.75 \\
& 2 & 221.25 & 103.5 & 324.75 \\
& 3 & 110.75 & 70 & 180.75 \\
& 4 & 217 & 134 & 351 \\
& 5 & 132 & 61.5 & 193.5 \\
& 6 & 155.5 & 102 & 257.5 \\
& 7 & 142 & 75.25 & 217.25 \\
& 8 & & & \\
& 1 & 206.5 & 153.8 & 360.3 \\
& 2 & 212 & 149 & 361 \\
& 3 & 145 & 182.2 & 327.2 \\
& 4 & 174 & 91 & 265 \\
& 5 & 205.5 & 121.75 & 327.25 \\
& 6 & & & \\
& 7 & 228.5 & 150.5 & 379 \\
& 8 & & & \\
\hline
\end{tabular}

${ }^{*}$ Number of minutes spent mursing during observation periods.

\begin{abstract}
Apparatus
The subjects were housed one per cage in $76 \times 76 \times 25 \mathrm{~cm}$ wood and wire mesh observation cages. Cage floors were marked off into $15 \times 15 \mathrm{~cm}$ squares. Lights in the observation room were on a 12-h day-night cycle, set at 7:30 am and pm. Five days preparturition, nest building material was provided.
\end{abstract}

\section{Procedure}

Rejection (defined as number of pups consumed), amount of time spent nursing, and activity (defined as number of floor squares crossed) were observed for $10 \mathrm{~min}$, four times per day at $8,11 \mathrm{am}$, and 2 , and $5 \mathrm{pm}$ for 2 weeks post partum, the period during which all normal females continue to engage in maternal behavior (Beach, 1937; Rosenblatt \& Lehrman, 1963; Stone, 1938). The size of the litters was manipulated to 4 to 8 pups per mother through randomly switching pups across groups so that each litter had control and DDT pups.

\section{RESULTS AND DISCUSSION}

Each group had an average of approximately five pups per litter. There was nonsignificant difference between the groups in litter size, $F(2,19)<1$.

A total of 16 pups was consumed by Group 50, and 5 each for Groups 0 and 100 . A chi-square analysis of these data revealed that significantly more pups were consumed by Group 50 and less than expected by Groups 0 and 100 based on the proportion of pups in each respective group to the total number of pups, $\chi^{2}(2)$ $=9.37, \mathrm{p}<.01$.

The loss of one subject in Group 100 and one subject in Group 50 led to a least-squares analysis (Kirk, 1968) of the nursing data since these subjects had consumed their pups immediately after birth. The analysis of the nursing data revealed a significant main effect of dosage $[F(2,18)=7.53, p<.01]$. Using Tukey's HSD test (Kirk, 1968) it was found that there was significantly less nursing by Group 50 (mean $=223.54$ ) than Groups 100 and 0 (means $=336.63$ and 362.5 , respectively), the latter two groups did not differ significantly. As is apparent in Table 1, nursing decreased for all subjects over weeks $[F(1,18)=81.15, p<.01]$.

The loss of one nonpregnant subject in Group 100 led to an unweighted means analysis of the activity data, and showed a significant increase in activity over weeks $[F(1,20)=14.54, p<.01]$. The pronounced increase in activity over successive days of Group 0 , apparent in Table 2 , seemingly washed out any cumulative DDT effect $[\mathrm{F}(2,20)=2.7, .05<\mathrm{p}<.10$.

The behavior of Group 50 appears to be a result of hyperexcitability induced by DDT (Goodman \& Gilman, 1970; Sobotka, 1971), and the data also indicate that very high concentrations of DDT (Group 100) cause prostration (O'Brien, 1967). This latter observation is the suggested explanation of the observed nonlinear relationship between dosage of DDT and activity. The activity for Group 50 was relatively high during Weeks 1 and 2, while the activity of Group 100 remained relatively low. Group 0 exhibited normal weaning behavior in that activity was low during the first week but gradually increased during Week 2 .

The data further suggest that the hyperexcitability

Table 2

Individual Activity* for Groups 0, 50, and 100 Over Weeks 1 and 2

\begin{tabular}{ccccr}
\hline DDT Dosage & Subject & Week 1 & Week 2 & Total \\
\hline & 1 & 218 & 202 & 420 \\
& 2 & 259 & 356 & 615 \\
$0 /$ ppm & 3 & 268 & 641 & 909 \\
& 4 & 318 & 1234 & 1552 \\
& 5 & 414 & 550 & 964 \\
& 6 & 373 & 488 & 861 \\
& 7 & 316 & 626 & 942 \\
& 8 & 130 & 808 & 938 \\
$50 /$ ppm & 1 & 249 & 256 & 505 \\
& 2 & 272 & 396 & 668 \\
& 3 & 369 & 349 & 718 \\
& 4 & 321 & 486 & 807 \\
& 5 & 619 & 836 & 1455 \\
& 7 & 1210 & 1588 & 2798 \\
& 8 & 729 & 451 & 1180 \\
& 1 & 446 & 325 & 571 \\
& 2 & 387 & 432 & 844 \\
& 2 & 508 & 895 \\
& 3 & 187 & 304 & 491 \\
& 4 & 338 & 487 & 825 \\
& 5 & 335 & 574 & 909 \\
& 6 & 109 & 178 & 287 \\
& 7 & 275 & 381 & 656 \\
& 8 & & & \\
\hline
\end{tabular}

*Defined as number of squares crossed. 
effect of DDT upon Group 50 contributed to significantly less nursing activity than in Groups 0 and 100. Also, Group 100, rather than being hyperexcitable, was prostrated by the DDT and thus remained in the nest and nursed.

Further evidence for the hyperexcitability-prostration interpretation is provided by the rejection data. Poor mothering by the animals in Group 50 led to the death of significantly more pups than expected based on the proportion of pups in Group 50 to the total number of pups. The switching of pups across litters renders an alternate interpretation of degree of pup DDT content as an explanation of pup consumption untenable. It should be mentioned that while many of the pups in Group 50 were allowed by the mothers to expire via nonnursing and nonretrieval, cannibalism of healthy pups was also observed.

In conclusion, the data of the present experiment would appear to support the hypothesis that poor maternal behavior, and not only direct ingestion of DDT, may cause harm to neonates.

\section{REFERENCES}

Al-Hachim, J. G., \& Fink, G. B. Effect of DDT or Parathion on open-field behavior of offspring from DDT- or Parathion-treated mothers. Psychological Reports, 1968, 22, 1193-1196.
Beach, F. A. The neural basis of innate behavior: I. Relative effects of cortical lesions upon the maternal behavior pattern in the rat. Journal of Comparative Psychology, 1937, 24, 393-440.

Fahim, M. S., Bennett, R., \& Hall, D. G. Effect of DDT on the nursing neonate. Nature, 1970, 228, 1222-1223.

Farney, D. New hope at Patuxent. National Wildlife, 1971, 9 , 45-47.

Goodman, L. S., \& Gilman, A. The pharmacological basis of therapeutics. New York: McMillan, 1970.

Henderson, G. L., \& Woolley, D. E. Studies on the relative insensitivity of the immature rat to the neurotoxic effects of 1, 1, 1, -Trichloro-2, 2-Bix (p-Chlorophenyl) ethane (DDT). Journal of Pharmacology \& Experimental Therapeutics, 1969 , $170,173-180$.

Kirk, R. E. Experimental design: Procedures for the behavioral sciences. Belmont, Cal: Brooks/Cole, 1968.

Lu, F. C., Jessup, D. C., \& Levallee, A. Toxicity of pesticides in young vs. adult rats. Food and Cosmetic Toxicology, 1965, 3, 591-596.

Naishtein, S. Y., \& Leibovich, D. L. Effect of small doses of DDT and lindane and their mixture on sexual function and embry ogenesis in rats. Gigiena I Sanitariia, 1971, 36, 190-195.

O'Brien, R. D. Insecticides: Action and metabolism. New York: Academic Press, 1967, Pp. 108-135.

Paulsen, K., Adesso, V. J., \& Porter, J. J. Report on pilot study of effect of DDT on maternal behavior. Bulletin of the Psychonomic Society, 1973, 1, 205-206.

Rosenblatt, J. S., \& Lehrman, D. D. Maternal behavior of the laboratory rat. In H. L. Rheingold (Ed.), Maternal behavior in mammals. New York: Wiley, 1963, Pp. 8-54.

Sobotka, T. J. Behavioral effects of low doses of DDT Proceedings of the Society for Experimental Biology \& Medicine, 1971, 137, 952-955.

Stone, C. P. Effects of cortical destriction on reproductive behavior and maze learning in albino rats. Journal of Comparative Psychology, 1938, 26, 217-236.

Treon, F. J., Boyd, J., Berryman, F., Gosney, J., Hartman, L., Brown, D., \& Coomer, J. Final report. Kettering Laboratory, University of Cincinnatti, College of Medicine, 1954.

(Received for publication November 14, 1974.)

\title{
Anagram solution times, word length, and type of accessory clue
}

\author{
D. J. MURRAY and L. L. MASTRONARDI \\ Queen's University, Kingston, Ontario, Canada
}

\begin{abstract}
Previous research on long-term memory has shown that the number of items retrieved is a power function of the number presented. An application of this power law to the prediction of anagram solution times as a function of word length was, in general, successful. It was also found that giving semantic cues decreased solution time more than did giving graphemic cues.
\end{abstract}

Studies on the retrieval of items such as words or pictures have shown, that, if $P$ items are presented, and $R$ are retricved, then

$$
\mathrm{R}=\mathrm{k} \mathrm{Pm}
$$

This paper is sponsored by P. C. Dodwell, who takes full editorial responsibility for it. where $\mathrm{k}$ and $\mathrm{m}$ are parameters to be found empirically (Standing, 1973; Murray, in press). The question asked in the present work was whether a similar formula could be applied in determining how long it takes to solve an anagram. It seems likely that in solving an anagram the subject processes a number of permutations of the letters of the anagram until he eventually finds a 